\title{
Design Parameters in Multimodal Games for Rehabilitation
}

\author{
Nauman Shah, BEng (Hons), Angelo Basteris, PhD, and Farshid Amirabdollahian, PhD
}

\begin{abstract}
Objectives: The repetitive and sometimes mundane nature of conventional rehabilitation therapy provides an ideal opportunity for development of interactive and challenging therapeutic games that have the potential to engage and motivate the players. Certain game design parameters that may encourage patients to actively participate by making the games more enjoyable have been identified. In this article, we describe a formative study in which we designed and evaluated some of these parameters with healthy subjects.

Materials and Methods: The "operant conditioning" and "scoring" design parameters were incorporated in a remake of a classic labyrinth game, "Marble Maze." A group of participants $(n=37)$ played the game twice: Once in the control condition without both modalities and then with either one of the parameters or with both. Measures of game duration and number of fails in the game were recorded along with survey questionnaires to measure player perceptions of intrinsic motivation on the game.

Results: Longer playtimes, higher levels of interest/enjoyment, and effort to play the game were recorded with the introduction of these parameters.

Conclusions: This study provides an understanding on how game design parameters can be used to motivate and encourage people to play longer. With these positive results, future aims are to test the parameters with stroke patients, providing much clearer insight as to what influences these parameters have on patients undergoing therapy. The ultimate goal is to utilize game design in order to maintain longer therapeutic interaction between a patient and his or her therapy medium.
\end{abstract}

\section{Introduction}

W Iтн Aвоut 795,000 victims in the United States ${ }^{1}$ and about 150,000 in the United Kingdom ${ }^{2}$ every year, stroke has become the leading cause of serious, long-term disability. With conventional exercise therapy being the only main effective treatment for stroke rehabilitation, the demand for rehabilitation services is very high and is likely to increase as the population ages. ${ }^{3}$ Along with diminishing resources to fund such rehabilitative care, it is difficult to provide an appropriate level of therapy to meet the patients' needs.

However, perhaps a more important reason to look for alternatives to conventional therapy is the monotonous nature of the exercises. It is not uncommon for stroke survivors to experience depression and anxiety following such a drastic change in lifestyle, and they may find it difficult to concentrate and actively participate in the therapy. Moreover, although repetitive exercises have shown to restore motor movements, ${ }^{4}$ it is also important for patients to actively participate in these exercises. ${ }^{5}$ The monotony of these exercises is often perceived as mundane and boring in nature by the patients, which poses a challenge to maintain patient motivation and engagement. ${ }^{6}$
In recent years, substantial work has been dedicated to innovate mechanisms that would improve the efficacy of rehabilitation process. Systems ranging from robotic devices 7,8 to provide physical support and movement assistance for patients performing the exercises to highly immersive virtual reality systems ${ }^{9}$ have been used for rehabilitative purposes. Generally, these systems work on the principle of providing the patients with an interactive user interface and implementing different task-oriented scenarios, allowing the patients to exercise in a systematic manner. Although these devices show a great potential as an alternative to conventional therapy ${ }^{10,11}$ and despite the existence of growing numbers of rehabilitation systems, studies have focused more on the hardware technologies of the system than the user interface. This gap represents a significant oversight in assessing the effectiveness and efficiency of the device as interface design and system usability can have an impact on the system's usefulness and efficacy.

Motivation is also an important aspect of post-stroke rehabilitation; however, it has rarely been investigated in stroke patients. ${ }^{12}$ Understandably, motivation brings patients to actively participate in the therapy. Furthermore, recent studies have shown that patients seem to exercise harder as a

Adaptive Systems Research Group, School of Computer Science, University of Hertfordshire, Hatfield, Hertfordshire, United Kingdom. 
result of more intense and interactive exercises. ${ }^{5}$ Acknowledging that participation is a prerequisite to increased treatment, it can be concluded that increases in sustained participation and adherence to the rehabilitation process will lead to greater recovery. ${ }^{13}$

Following the belief that rehabilitation exercises and activities that are of interest to the patients motivate them to sustain or increase performance, ${ }^{14}$ recent studies have focused their attention on using interactive game-like interfaces for their systems. Although a few prototypes of this type of systems exist, games remain simplistic and often underevaluated in terms of effectiveness and appropriateness for the rehabilitation process ${ }^{15}$ owing to needs to reduce the cognitive load on patients. Although some of the current systems use games for human-robot/human-computer interactions, most of these games are not designed to be entertaining or motivational for the patient but are only used to present the exercise tasks in a more visual and multimodal manner. Hence the question remains regarding their ability to sustain the patients' attention and interest during the exercise.

This study aims at understanding how the game design techniques affect the motivation of the players and the gameplay for rehabilitation games. Several hypotheses are tested using specific subscales of Intrinsic Motivation Inventory (IMI) studies. ${ }^{16,17}$ We hypothesize that the addition of operant conditioning parameters (based on rewards and challenges given to the players according to their performance [more details are given in Materials and Methods]) will facilitate experiences of enjoyment and effort in gameplay. We also incorporate scoring and feedback mechanisms that we expect to be associated with game competence and importance. We further suggest that the combination of these parameters facilitates players' motivation, high user engagement, and longer play time, as assessed by the rated enjoyment and competence through which we will assess changes from before to after gameplay. This preliminary work is associated with an ongoing European Commission-funded project, Supervised Care and Rehabilitation Involving Personal Tele-Robotics (SCRIPT), aimed at exploiting these findings in rehabilitation of stroke subjects. The games devised and tested here are considered within the project for exercise at home at chronic (6 months plus) stages of stroke.

\section{Materials and Methods}

\section{Study design}

This study is an initial test of the design features on a general sample of healthy participants. Games considered in this study will undergo a four-stage evaluation. At first, designed games pass a formative evaluation with healthy volunteers. The resultant game is then evaluated using a large group of participants. This article provides the results from this stage. Our future work focuses on incorporating results obtained from this summative evaluation, and further formative evaluation to be conducted with stroke patients, toward a long-term summative evaluation with stroke patients in their homes. This is thought to ultimately ascertain usefulness of these games and their parameters in sustaining long-term engagement during therapeutic interaction facilitated by these games.

This study has been approved by the University of Hertfordshire's ethics board (protocol number COM/PG/UH/ 00001).

\section{Intervention}

Because the primary concern of the study is with upper limb stroke rehabilitation, we developed a prototype game that aims to exercise patients' affected wrist using flexion/ extension and pronation/supination movements and hand grasping motions. This aims at achieving fine motor skills of the wrist and hand. The game, called "The Marble Maze," is a remake of the classic labyrinth game where the user is shown a marble in a maze (Fig. 1). The player has to guide the marble to a goal location, avoiding holes on the way through which

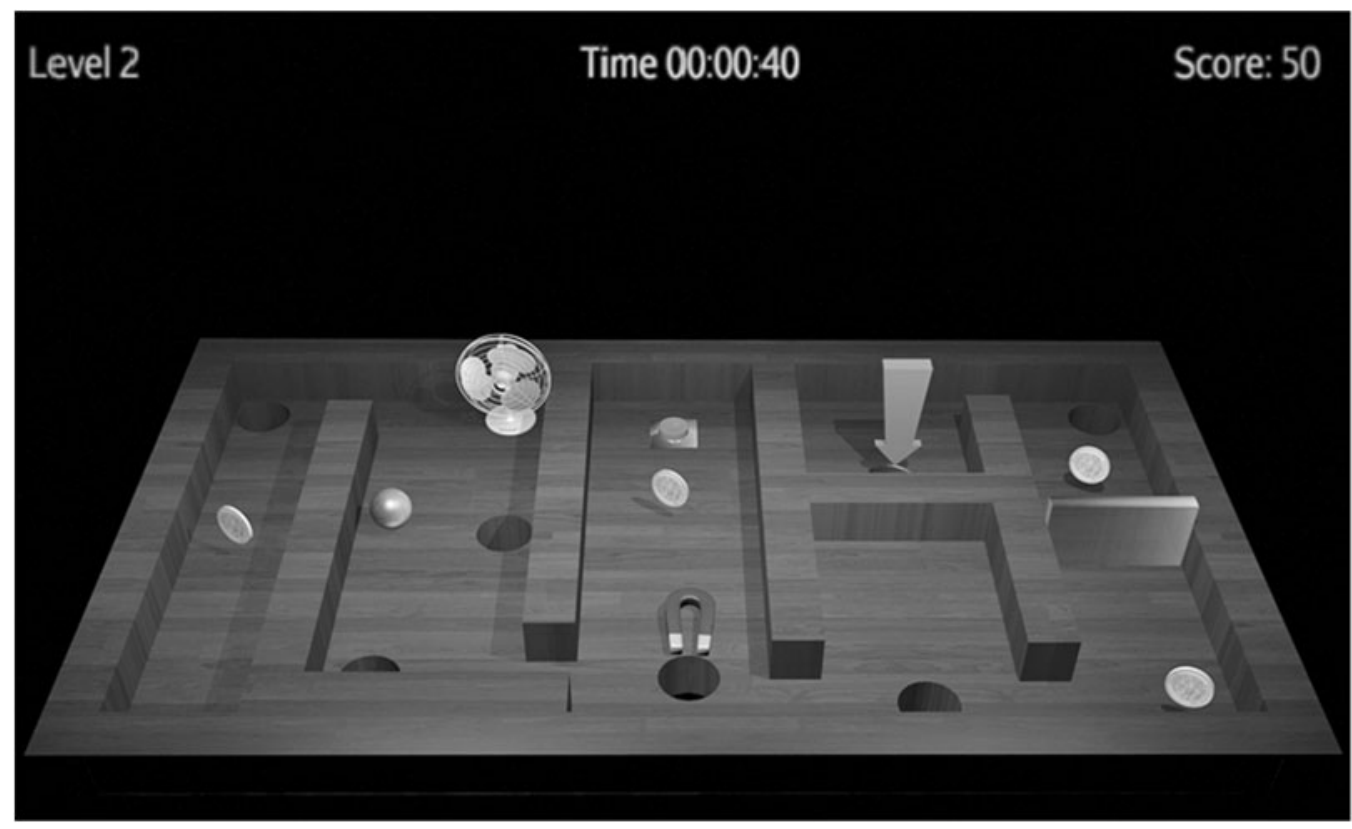

FIG. 1. Screenshot of the "Marble Maze" game. 
the marble could fall down. The player controls the marble by tilting the lightweight polystyrene (Styrofoam ${ }^{\circledR}$; Dow Chemical Co., Midland, MI) board, which makes the marble roll in accordance with the orientation of the maze. The orientation of the maze is directly mapped to a Styrofoam board attached to a Geomagic ${ }^{\circledR}$ Touch $^{\mathrm{TM}}$ haptic device (formerly known as the Sensable Phantom Omni; Geomagic, Morrisville, NC) (Fig. 2) held by the player, hence providing a feel of actually holding the virtual maze on the screen. The aim of the game is to guide the marble successfully to the goal before the time runs out. If the marble falls in a hole or the timer runs out, the game restarts with the marble back to its starting position.

The game models were designed in Blender and programmed on the Unity3D game engine by the authors. The experiment was carried out on a Windows 7 Professional operating system.

In addition to this, some of the game design techniques used by commercial game developers ${ }^{18-22}$ were incorporated in the game with the aim to see how each of these parameters affects the motivation of the players:

1. Beating the game. To incorporate this, five different stages of the game were made. Each stage presented the player with a different maze. As the player gained command over the controls and the game progressed, the stages of the game unlocked, one after the other. Each stage was designed to have a higher difficulty level than the previous ones. For example, increased complexity of the maze, lesser time to complete, or more holes to dodge were used to increase difficulty.

2. Scoring. A scoring mechanism was built into the game to motivate the player in performing better. The sooner the player guides the marble to the goal, the higher the score. In addition, virtual coins placed in the maze could be collected to achieve bonus scores. Some coins were placed outside the shortest path to the goal, and the choice was left to the player either to collect the coins for extra score or simply to finish the stage to go on to the next.

3. Operant conditioning. This technique is built on the principle of incorporating within the game a fixed ratio

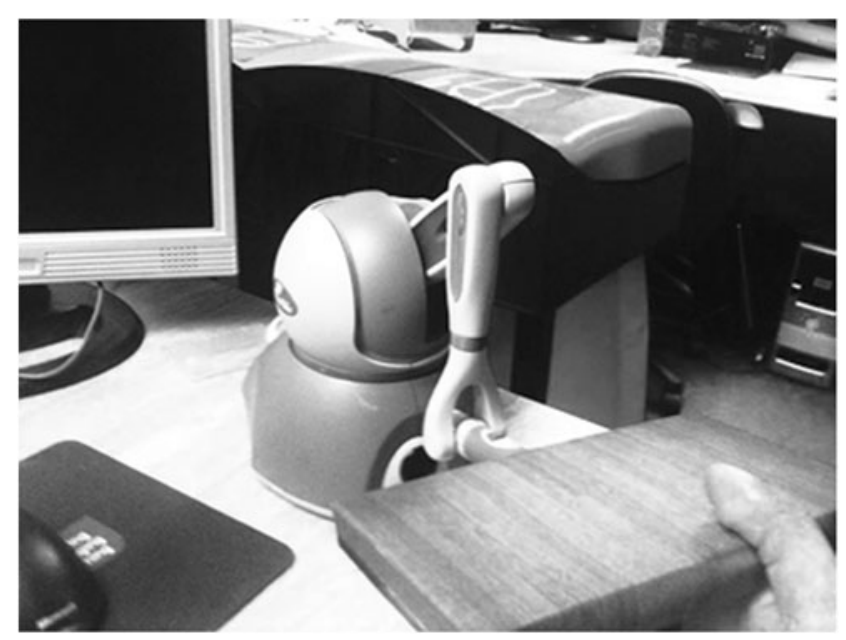

FIG. 2. The Phantom Omni with Styrofoam board extension. schedule of reinforcements and aversive stimuli for players to avoid. In addition to the bonus score for each of the coins obtained by the user, certain "power-ups" or rewards were built in the game. These power-ups were obtained by collecting three coins in a row in a single stage. For every third coin collected, the player either gained double score for the third coin, an extra 30 or 15 seconds added to the timer to complete the stage, or an ability to go through a wall of the maze. The choice of these rewards was random to build an element of surprise for the player. In addition to the rewards, small punishments or challenges were also built in to make the game more challenging. In each of the stages, small obstacles were placed to deviate the path of the marble, requiring the player to think over which path was secure to take. These obstacles included magnets, which would activate when the marble came close to the magnet and would pull the marble toward itself. Another obstacle was a small fan, which would blow the marble away as it came near, usually toward a hole or making it harder to collect a coin. A final obstacle was a door that would block the path to the goal location and could be opened only by pressing a red button placed somewhere in the maze.

4. Feedback. At the end of each stage, a screen was designed to display the player's performance. It included the score achieved in the stage, the time taken, and the number of times the marble fell. The screen also gave the players the chance to replay a stage in the case they were not satisfied with their performance.

In order to observe the effects of these parameters we developed four versions of the game:

- Basic version. The basic version consisted of neither the operant conditioning nor the scoring parameters. No score was recorded, and the player simply had to complete the stage to move on to the next.

- Operant conditioning version (OC version). This version had the rewards and challenges of the operant conditioning parameter built in. However, no score was recorded.

- Scoring version (SC version). This version had the scoring mechanisms turned on without the operant conditioning.

- Operant conditioning + scoring version (OC-SC version). This version had both the operant conditioning and the scoring parameters built in.

\section{Experimental settings}

During 4 days in 2012, participants were recruited among people who called at an installation in a public venue of the University of Hertfordshire (Hatfield, Herts, United Kingdom). Preliminary explanation of the research and its aims was given to people as they came along, eventually in groups. Those who showed interest in taking part filled in a consent form. The participants were then briefed about the structure of the session and how to play the game and then participated to a 10-15-minute session that was administered by the first author. After playing each session, they were asked to fill in a questionnaire. They would then play the alternate version and were asked to fill in the survey once again. 
Participants were assigned to one of the three groups: OC, SC, or OC-SC. Each participant played two versions of the game: the basic version and another, depending on the randomized group to which they were assigned. The version played first among the two was also randomly decided for each participant as counterbalancing. As the switching between experimental versions of the game took time (order of minutes), if participants were recruited jointly they had been assigned to the same experimental group, thus allowing the waiting time to be kept to a minimum. However, care was taken so that the experiment would have then been conducted separately, with each subject not being exposed to the previous participant's briefing gameplay. This allocation resulted in a slight imbalance in the number of participants per group as highlighted below.

Once the game started, they could pause the game any time or stop the session if they felt any discomfort or did not prefer to continue.

Thirty-seven participants took part in the study (26 males and 11 females; mean age, 21.6 years; standard deviation, \pm 2.3 ). Because the participation was voluntary, assignment to the groups was randomized, and no incentive was offered, an equal number of participants could not be achieved (OC, $n=13$; SC, $n=11$; OC-SC, $n=13$ ).

Outcome measures. As a primary outcome measure, survey questionnaires were adapted from the IMI to measure players' perceptions of intrinsic motivation on the game. The IMI is a multidimensional measurement instrument that contains several subscales. Five question categories were included in a questionnaire: Interest/enjoyment, perceived competence, effort/importance, pressure/tension (reversed), and perceived choice (Table 1). Each question category consisted of five questions with answers ranging from 1 to 7 , with results from each category summed up for analysis. Thus a total score of 35 was the maximum achievable total for each category. The reliability of the IMI and its validity had been established by previous studies, ${ }^{16,17}$ although they had some variances in the context and languages compared with this study.

Additionally, the secondary outcome measurements include the gameplay duration and the number of failures (i.e., the marble fell into the wrong hole). These quantities were compared for the OC, SC, and OC-SC versions of the game relative to the basic version.

Data analysis. The differences in each category between basic and experimental (OC, SC, and OC-SC) versions of the game were determined by using repeated-measures analysis of variance. A paired-sample $t$ test was conducted to compare the means of the time taken to complete the different versions of the game between the basic and the experimental versions. In order to compare the medians of the number of fails among the basic and the experimental versions, a Wilcoxon signedtest was used as a nonparametric analytical method.

These analyses were performed using SPSS Statistics version 21.0 software (IBM, Armonk, NY).

\section{Results}

Results obtained during the experiment are divided into observations based on different outcome measures used in this study.

\section{IMI scores for each group}

Game versions with the operant conditioning parameters and/or the scoring parameters led to higher scores for enjoyment/interest $(P<0.0005)$, competence $(P=0.002)$, and effort/importance $(P<0.0005)$, compared with the versions

Table 1. Survey Questionnaire

\begin{tabular}{|c|c|c|}
\hline Number & Category & Question \\
\hline 1 & Enjoyment/interest & When I was playing the game, I was thinking about how much I enjoyed it. \\
\hline 2 & & I would describe the game as very enjoyable. \\
\hline 3 & & I thought the game was very boring. \\
\hline 4 & & I had fun playing the game. \\
\hline 5 & & The game did not hold my attention. \\
\hline 6 & Perceived choice & I felt like I was doing what I wanted while playing the game. \\
\hline 7 & & I felt in control of how to play the game. \\
\hline 8 & & I did not have a lot of choice about how to do the in-game tasks. \\
\hline 9 & & I did things because they interested me. \\
\hline 10 & & I felt controlled and pressured in a certain way. \\
\hline 11 & Pressure/tension & I felt very capable and effective. \\
\hline 12 & & The game was too difficult for me. \\
\hline 13 & & The game kept me on my toes but did not overwhelm me. \\
\hline 14 & & I was frustrated while playing the game. \\
\hline 15 & & I felt relaxed while playing the game. \\
\hline 16 & Perceived competence & I was satisfied by my performance. \\
\hline 17 & & I feel pretty skilled at the game. \\
\hline 18 & & After playing the game for a while, I felt very competent. \\
\hline 19 & & I would like to play the game again. \\
\hline 20 & & I want to perform better than others in the game. \\
\hline 21 & Effort/importance & I put in a lot of effort to play this game. \\
\hline 22 & & I tried hard to do well in the game. \\
\hline 23 & & It was not important for me to do well in the game. \\
\hline 24 & & It was important for me to do well in the game. \\
\hline 25 & & I did not try to do well in the game. \\
\hline
\end{tabular}



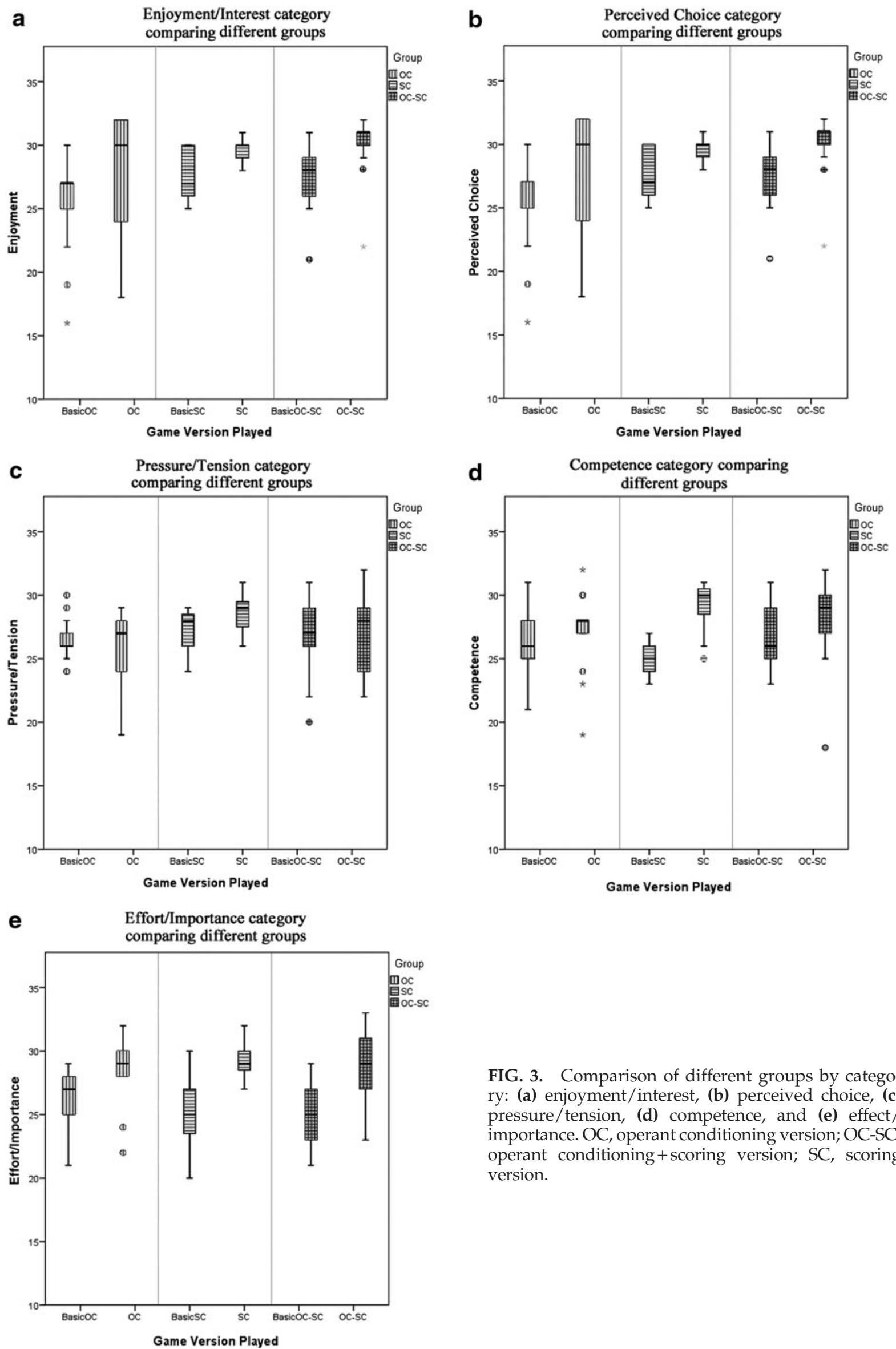

FIG. 3. Comparison of different groups by category: (a) enjoyment/interest, (b) perceived choice, (c) pressure/tension, (d) competence, and (e) effect/ importance. OC, operant conditioning version; OC-SC, operant conditioning + scoring version; SC, scoring version. 
without these parameters, whereas addition of these parameters did not affect perceived choice $(P=0.066)$ or pressure/ tension $(P=0.379)$.

Figure 3 shows the IMI scores for the different group medians in the boxes with outlier values as circles and stars for (a) enjoyment/interest, (b) perceived choice, (c) pressure/ tension, (d) competence, and (e) effort/importance categories.

\section{Comparisons between time played and number of fails}

There was a statistically significant difference reflected by the duration between the basic version and the OC version as determined by paired-sample $t$ test among the basic version and the $\mathrm{OC}$ version $(P=0.002)$. However, there was no statistically significant difference in the number of fails between the basic version and the OC version as determined by the Wilcoxon signed-rank test $(P=0.418)$.

A paired-sample $t$ test showed no difference in the duration of play for the basic version and the SC version $(P=0.189)$. However, the number of fails in this group differed significantly between the basic version and the SC version as determined by the Wilcoxon signed-rank test $(P=0.04)$.

A paired-sample $t$ test showed an increase in the time taken to complete the basic version and the OC-SC version $(P<0.005)$. Also, there was a statistically significant difference in the number of fails between the basic version and the OC$\mathrm{SC}$ version as determined by the Wilcoxon signed-rank test $(P=0.045)$.

Figure 4 presents a comparison for the duration of play between different experimental conditions (Fig. 4a) while providing the comparative value on number of fails encountered by the experimental groups (Fig. 4b). Figure 4 allows for comparing between basic version conditions and the OC, SC, and OC-SC version conditions with the appropriate pairing.

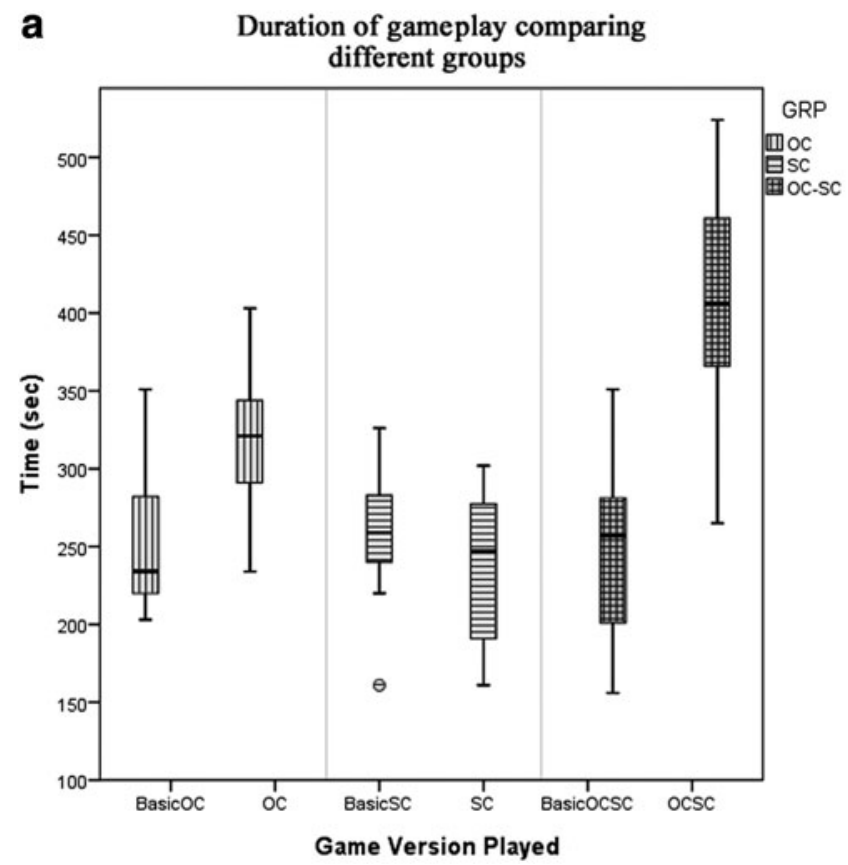

\section{Discussion}

The objective of this study was to test some key game design parameters and investigate their effects on player motivation, enjoyment, and gameplay times. These parameters, once identified, can be incorporated into rehabilitation games in order to motivate and engage patients undergoing therapy into participating in their exercise regimes more actively.

The results obtained from this study suggested that addition of both parameters-operant conditioning and scoringresults in higher motivational scores.

The results also suggested that although the operant conditioning parameter had no effect on the number of fails, the time taken to complete the game was significantly increased because of it. This was perhaps because of the time taken to solve or overcome the challenges introduced by the operant conditioning parameter. Also, the fact that for every third coin collected the player receives a bonus makes him or her keen on deviating from the shortest possible path, taking longer to complete the game. Because there was no score, the players were not under any pressure to complete the game quickly, giving them plenty of time to maneuver carefully, hence the lower number of fails. This could, however, be different from stroke patients, where fine motor control might be lacking.

In contrast, when the scoring parameter was introduced, the participants tended to finish the game quickly in order to achieve higher scores within shorter playtime. Although the players were under pressure to complete the game quickly, the lack of additional challenges (as there were in the OC version) resulted in a lower number of fails.

It is interesting that we observed that the operant conditioning parameter increased the playtime (as shown in Fig. $4 a)$, but it affected the number of fails only when there was a

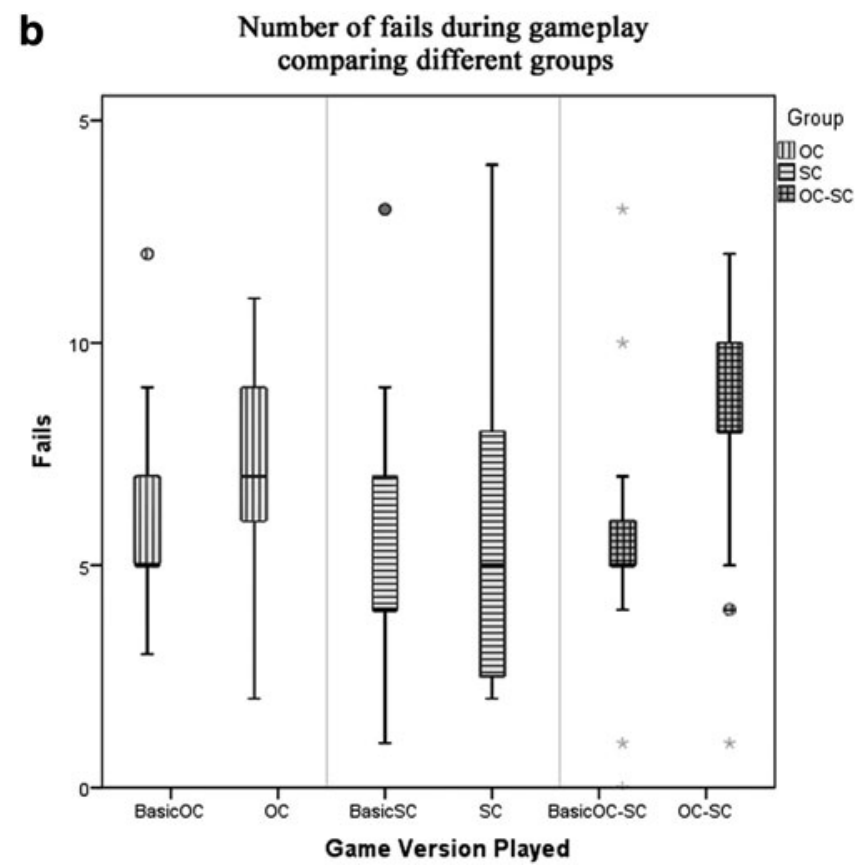

FIG. 4. (a) Duration of and (b) number of fails during gameplay comparing different groups: operant conditioning version (OC), scoring version (SC), and operant conditioning + scoring version (OC-SC). 
scoring mechanism. Similarly, the scoring parameter on its own decreased the number of failures. The incorporation of both differed from their individual effects. We observed that the number of failures and the playtimes had both increased significantly (Fig. 4a) without compromising the player's enjoyment (Fig. 3a). This might be because the additional challenges added by the operant conditioning parameter may be easier to overcome when there is no pressure to complete the game quickly, unlike when put together with a score. This led to an important conclusion that it is not only the parameters that are important, but also the combination of the different parameters together that results in player motivation and longer gameplays.

Because this study was an initial test of the design parameters, it was carried out only with healthy participants, and it is thus not possible to conclude if these parameters are beneficial in rehabilitation games. However, the results suggested that the key parameters tested could be used to influence patient motivations and increase game playtime among rehabilitative games, especially if aligned with rehabilitation objectives. Also, the game is only suitable for patients with some wrist movement, and a certain level of cognitive skills is required to comprehend the flow of the game.

Because videogames are highly associated with user engagement, the idea of using games for motivating patients undergoing therapy is very appealing. Literature has shown that integration of games with a virtual environment helps to enhance the motivation of people undergoing therapy. ${ }^{23} \mathrm{~A}$ study carried out at the U.S Army Research Institute for Behavioural and Social Sciences reported on the basis of a presence questionnaire and immersive tendencies questionnaires that a multimodal virtual environment with rich graphical models can be designed to engage, immerse, and motivate people. ${ }^{24}$

Some research studies have examined the potential of existing commercial games and consoles for stroke rehabilitation. ${ }^{25,26}$ Although these games are rich with design features, they are not often suitable for patients with limited motor abilities. For this reason, the rehabilitation games need to be designed specifically for the purpose and be customizable according to each individual's needs.

Few recent research studies have focused on developing and testing systems designed specifically for stroke patients. Burke et al. ${ }^{27}$ have designed and tested games specifically made for stroke rehabilitation and concluded that welldesigned games can be highly engaging and, if they promote limb motion, can be beneficial for the rehabilitation. Alankus et al. ${ }^{28}$ also designed and tested games specific for stroke patients and reported similar findings based on a more qualitative approach. Although these games may be beneficial already, they lack some game design parameters the commercial game designers use, which we believe can be improved.

\section{Future Work}

We have made progress toward understanding how game design parameters can be used to motivate and encourage healthy participants to play longer. With these positive results presented, we now aim to test games with these parameters with stroke patients, which would provide a much clearer insight to what influence these parameters have on patients undergoing therapy when compared with the healthy subjects. The SCRIPT project focuses on hand and wrist stroke therapy utilized by either a passive or an active exoskeleton device, linking hand and finger movements to therapeutic games. During our evaluation, patients can choose freely different games at the comfort of their homes. Our intention is to utilize our results here in order to provide a medium for interaction between hand/wrist motion and performing some selected games; thus we hope to provide a chance for a sustained interest in a home-based rehabilitation system. We intend to utilize these results, alongside two more stages of work planned to evaluate, formatively (during design) and summatively (as a complete post-design evaluation), the use and benefits of these games. Additional games developed by the authors, as well as games developed by our project partners, will provide a larger number of choices, aiming to appeal to a wider spectrum of patient preferences.

\section{Author Disclosure Statement}

No competing financial interests exist.

\section{References}

1. Go AS, Mozaffarian D, Roger VL, et al. Heart disease and stroke statistics-2013 update: A report from the American Heart Association. Circulation 2013; 127:e6-e245. Erratum in: Circulation 2013; 127:e841.

2. Stroke statistics update-18 December 2012. www.isdscotland .org/Health-Topics/Stroke/Publications (accessed December 13, 2013).

3. Foerch C, Sitzer M, Steinmetz H, Neumann-Haefelin T. Future demographic trends decrease the proportion of ischemic stroke patients receiving thrombolytic therapy: A call to set-up therapeutic studies in the very old. Stroke 2009; 40:1900-1902.

4. Van Der Lee JH. Constraint-induced therapy for stroke: More of the same or something completely different? Curr Opin Neurol 2001; 14:741-744.

5. Loureiro RCV, Lamperd B, Collin C, Harwin WS. Reach \& grasp therapy: Effects of the Gentle/G system assessing sub-acute stroke whole-arm rehabilitation. In: 2009 IEEE International Conference on Rehabilitation Robotics. Piscataway, NJ: IEEE; 2009: 755-760.

6. Burke JW, Morrow PJ, McNeill MDJ, et al. Vision based games for upper-limb stroke rehabilitation. In: 2008 International Machine Vision and Image Processing Conference. Portrush, Northern Ireland. 3-5 Sept. 2008, pp. 159-164.

7. Amirabdollahian F. Rehabilitation robotics. In: Dautenhahn K, Saunders J, eds. Advances in Interaction Studies: New Frontiers in Human-Robot Interaction. Philadelphia: John Benjamins Publishing Co., 2011: 305-326.

8. Brewer BR, McDowell SK, Worthen-Chaudhari LC. Poststroke upper extremity rehabilitation: A review of robotic systems and clinical results. Top Stroke Rehabil 2007; 14:22-44.

9. Saposnik G, Levin M. Virtual reality in stroke rehabilitation: A meta-analysis and implications for clinicians. Stroke 2011; 42:1380-1386.

10. Prange GB, Jannink MJA, Groothuis-Oudshoorn CGM, et al. Systematic review of the effect of robot-aided therapy on recovery of the hemiparetic arm after stroke. J Rehabil Res Dev 2009; 43171-184.

11. Lo AC, Guarino PD, Richards LG, et al. Robot-assisted therapy for long-term upper-limb impairment after stroke. N Engl J Med 2010; 362:1772-1783. 
12. Maclean N. Qualitative analysis of stroke patients' motivation for rehabilitation. BMJ 2000; 321:1051-1054.

13. Maclean N, Pound P, Wolfe C, Rudd A. The concept of patient motivation: A qualitative analysis of stroke professionals' attitudes. Stroke 2002; 33:444-448.

14. Florey LL. Intrinsic motivation: The dynamics of occupational therapy theory. Am J Occup Ther 1969; 23:319-322.

15. Flores E, Tobon G, Cavallaro E, et al. Improving patient motivation in game development for motor deficit rehabilitation. In: International Conference on Advances in Computer Entertainment Technology ACE 2008, Yokohama, Japan. 3-5 December 2008, 381.

16. McAuley E, Duncan T, Tammen VV. Psychometric properties of the Intrinsic Motivation Inventory in a competitive sport setting: A confirmatory factor analysis. Res Q Exerc Sport 1989; 60:48-58.

17. Tsigilis $\mathrm{N}$, Theodosiou A. Temporal stability of the intrinsic motivation inventory. Percept Mot Skills 2003; 97:271-280.

18. van Vliet PM, Wulf G. Extrinsic feedback for motor learning after stroke: What is the evidence? Disabil Rehabil 2006; 28:831-840.

19. Nevid JS. Essentials of Psychology: Concepts and Applications. Chester Springs, PA: Cengage Learning; 2011: 521.

20. Griffiths M. Fruit machine gambling: The importance of structural characteristics. J Gambling Stud 1993; 9:101-120.

21. What makes a video game addictive? www.video-gameaddiction.org/what-makes-games-addictive.html (accessed February 3, 2012).

22. Bull-Hansen C. Serious Games: Video Game Design Techniques for Academic and Commercial Communication. Oslo: University of Oslo; 2007.
23. Rizzo A, Kim GJ. A SWOT analysis of the field of virtual reality rehabilitation and therapy. Presence 2005; 14:119146.

24. Witmer BG, Singer MJ. Measuring presence in virtual environments: A presence questionnaire. Presence 1998; 7:225240.

25. Saposnik G, Teasell R, Mamdani M, et al. Effectiveness of virtual reality using Wii gaming technology in stroke rehabilitation: A pilot randomized clinical trial and proof of principle. Stroke 2010; 41:1477-1484.

26. Yavuzer G, Senel A, Atay MB, Stam HJ. "Playstation EyeToy games" improve upper extremity-related motor functioning in subacute stroke: A randomized controlled clinical trial. Eur J Phys Rehabil Med 2008; 44:237-244.

27. Burke JW, McNeill MDJ, Charles DK, et al. Optimising engagement for stroke rehabilitation using serious games. Vis Comput 2009; 25:1085-1099.

28. Alankus G, Lazar A, May M, Kelleher C. Towards customizable games for stroke rehabilitation. In: ACM International Conference on Human Factors in Computing Systems CHI 2010, Atlanta, GA, USA. 10-15 April 2010, 2113.

Address correspondence to: Nauman Shah

Adaptive Systems Research Group School of Computer Science University of Hertfordshire Hatfield, AL10 9AB, United Kingdom

E-mail: nauman.shah@hotmail.co.uk 